\title{
Non-functioning Adrenocortical Carcinoma Arising in an Adrenal Rest: Immunohistochemical Study of an Adult Patient
}

\author{
Hiroshi Yokoyama, ${ }^{1}$ Tomotaro Adachi, ${ }^{1}$ Kazuna Tsubouchi, ${ }^{1}$ Masatoshi Tanaka ${ }^{1}$ \\ and Hironobu Sasano ${ }^{2}$ \\ ${ }^{1}$ Department of Urology, Faculty of Medicine, Fukuoka University, Fukuoka City, Fukuoka, Japan \\ ${ }^{2}$ Department of Pathology, Tohoku University School of Medicine, Sendai City, Miyagi, Japan
}

Adrenal rest is uncommon in adults, and usually represents a small lesion incidentally detected during surgery or autopsy. The adrenal rest can be detected anywhere along the path of embryonic migration of adrenal cortex, including celiac axis, genitals and broad ligament, and may be formed with the separation of cortical fragments by the migration of medullary elements from the sympathochromaffin tissue into the preformed cortical primordium. In addition, even primary adrenocortical carcinoma is a rare tumor with incidence 0.5-2 per million annually; therefore, adrenocortical carcinoma arising in adrenal rests is extremely rare. We encountered a patient with non-functioning ectopic adrenocortical carcinoma in retroperitoneum. A 34-year-old female presented with an incidentally discovered retroperitoneal mass revealed by abdominal ultrasound in her regular health examinations. She did not have any clinical abnormalities and underwent hand-assisted laparoscopic resection of the tumor. A dark-brown tumor, measuring $65 \times 56 \times 45 \mathrm{~mm}$, was identified in the retroperitoneal space between lower pole of right kidney and inferior vena cava. Histologically, the tumor was predominantly composed of compact eosinophilic cells forming nest-like arrangements and diffusely positive for the steroidogenic factor- 1 . The tumor met four of the criteria of Weiss used in histological diagnosis of adrenocortical carcinoma (eosinophillic cytoplasm, nuclear atypia, atypical mitosis, and sinusoidal invasion). The tumor cells were immunohistochemically positive for $17 \alpha$-hydroxylase, dehydroepiandrosterone sulfotransferase and $3 \beta$-hydroxysteroid dehydrogenase, each of which is involved in the synthesis of adrenocortical steroids. Therefore, based on these findings, we diagnosed this tumor as ectopic adrenocortical carcinoma arising in adrenal rest of retroperitoneum.

Keywords: adrenal rest; adrenocortical carcinoma; immunohistochemistry; pathology; steroidogenesis Tohoku J. Exp. Med., 2013 April, 229 (4), 267-270. C 2013 Tohoku University Medical Press

\section{Introduction}

Adrenal rests have been postulated to be formed by the separation of cortical fragments displaced by the migration of medullary elements from the sympathochromaffin tissue into the preformed cortical primordium (Goren et al. 1991). The incidence of adrenal rests could be as high as $50 \%$ in children, but the lesion generally disappears within a few years of birth (Goren et al. 1991).

Adrenal rests tumor are further uncommon and the great majority of clinically detected adrenal rest tumors are reported to be functioning (Goren et al. 1991). Nonfunctioning adrenal rest tumors are less frequent and were discovered incidentally (Goren et al. 1991). In addition, adrenocortical carcinoma is a rare tumor with incidence 0.5-2 per million population annually, and also an aggressive cancer with a poor prognosis with $32-45 \% 5$-year over- all survival (Icard et al. 2001). Therefore, adrenocortical carcinoma presumed to be arising in adrenal rest is extremely rare (Goren et al. 1991; Arai et al. 2000; Akishima-Fukasawa et al. 2011).

In this study, we herein report clinical aspects in the patient with non-functioning ectopic adrenocortical carcinoma arising in adrenal rest and its histopahtological findings including immunohistochemical evaluation.

\section{Clinical Report}

A 34-year-old female was presented to our institution with incidentally discovered retroperitoneal tumor following abdominal ultrasound in her regular health evaluation. An abdominal ultrasound imaging revealed a $6.5 \mathrm{~cm}$ right retroperitoneal mass between right kidney and inferior vena cava. Computed tomography scans of abdomen identified normal bilateral adrenal glands at normal location.

Received January 30, 2013; revised and accepted March 14, 2013. Published online April 19, 2013; doi: 10.1620/tjem.229.267.

Correspondence: Hiroshi Yokoyama, M.D., Ph.D., Department of Urology, Faculty of Medicine, Fukuoka University, 7-45-1 Nanakuma, Jonan-ku, Fukuoka 814-0180, Japan.

e-mail: hyokoyama@yokoyama-hp.or.jp 


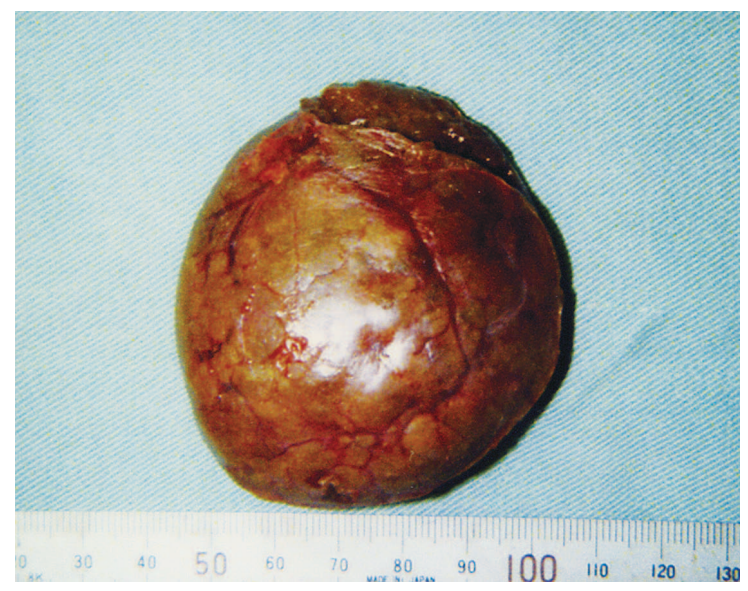

Fig. 1. Macroscopic appearance of the tumor. The tumor is well encapsulated and not accompanied by both hemorrhage and necrosis.

Laboratory evaluation of the patient's blood and 24-hours urine samples revealed normal adrenal functions.

A hand assisted laparoscopic resection of right retroperitoneal tumor was performed. The tumor was not adhered to right kidney and surrounding tissue and completely removed without any further complications. The dark-brown tumor, measuring $65 \times 56 \times 45 \mathrm{~mm}$, weighing 71 $\mathrm{g}$, was well encapsulated and did not show foci of hemorrhage and necrosis (Fig. 1).

Light microscopically, the tumor was composed predominantly of eosinophilic compact cells with nest-like formation (Fig. 2). There were not any areas simulating the non-pathological adrenal glands. The tumor was surrounded by a relatively dense fibrous capsule, and myelolipomatous changes were also detected in the tumor. These findings were histopathologically consistent with those of adrenocortical neoplasm. Among the criteria of Weiss for adrenocortical malignancy (Weiss 1984), factors on eosinophillic cytoplasm, nuclear atypia, atypical mitosis and sinusoidal invasion were met in the tumor, but those on capsular invasion, venous invasion, confluent necrosis, mitosis and architecture were not detected.

Immunohistochemically, almost all of the tumor cells were diffusely and strongly positive for steroidogenic factor-1 (SF-1) suggesting that these cells had the characteristic of adrenocortical cells (Fig. 3A). Based on the criteria of Weiss and the results of SF-1 immunohistochemistry, this lesion was diagnosed as adrenocortical carcinoma arising in ectopic adrenal gland located in the retroperitoneum. In addition, positive immunoreactivity for the three types of steroidogenic enzymes was detected in those cells. The relative immunointensity of these steroidogenic enzymes varied among tumor cells. Immunoreactivity for $17 \alpha-$ hydroxylase (P450c17) was mostly detected in the tumor cells (Fig. 3B), and immunoreactivity for dehydroepiandrosterone sulfotransferase (DHEA-ST) was partially detected in those (Fig. 3C). However, immunoreactivity for

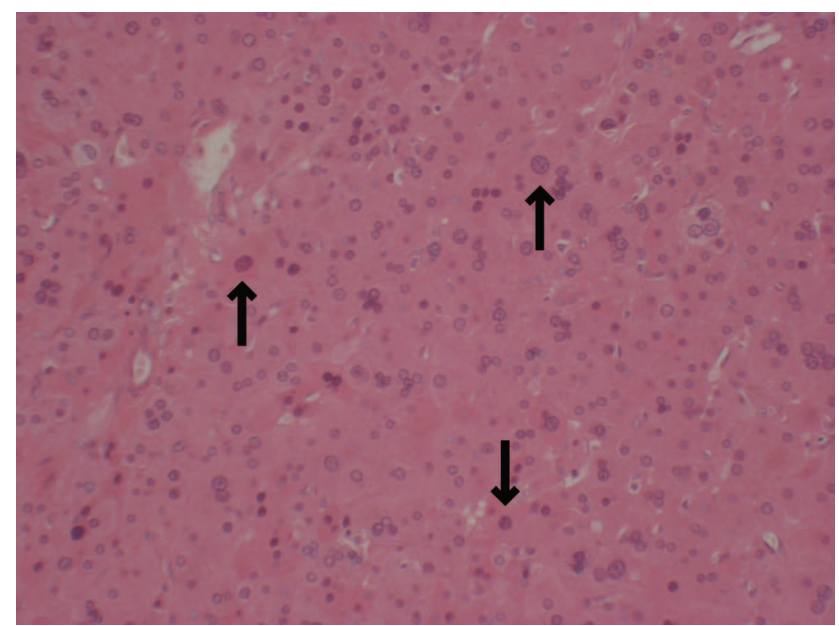

Fig. 2. Light microscopic features of the tumor with an original magnification $\times 200$. The tumor consisted predominantly of eosinophilic compact cells arranging nest-like formation. Arrows represent the characteristics features of tumor cells, i.e., relatively large nuclei with abundant eosinophilic cytoplasm.

$3 \beta$-hydroxysteroid dehydrogenase ( $3 \beta$-HSD) was rarely detected in these tumor cells (Fig. 3D). The control sections for negative immunostaining were performed using normal rabbit IgG or 0.01 M PBS instead of primary antibodies, and no specific immunoreactivity was detected in these sections (data not shown). Ki67 labeling index as a cellular marker to determine for proliferation was less than $1 \%$.

The postoperative course was unremarkable, and the patient has been clinically free from the disease after ten years of follow-up.

\section{Discussion}

Adrenal rest is uncommon in adults, and usually represents a minute lesion incidentally detected during surgery or autopsy (Goren et al. 1991; Arai et al. 2000). The rest may be detected anywhere along the path of embryonic migration including celiac axis, genitals and broad ligament, but the adrenal rest tumor is rare even in these areas (Arai et al. 2000).

In our patient, the tumor demonstrated light microscopic histopathological features similar to those of primary adrenocortical tumor. Weiss's criteria are generally used in distinguishing adrenocortical malignancy from benign tumors (Weiss 1984). The Ki67 labeling index represents an immunohistochemical marker for proliferation, and the mutations in the p53 tumor suppressor gene were also reported as another approach to differentiate between benign and malignant adrenocortical neoplasms (Arola et al. 2000). Although Ki67 labeling index in our patient was low with less than $1 \%$, the tumor had four positive points of Weiss's criteria. Therefore we considered this tumor as adrenocortical carcimona. However it is important to point out that due to the rarity of adrenocortical tumor arising in 


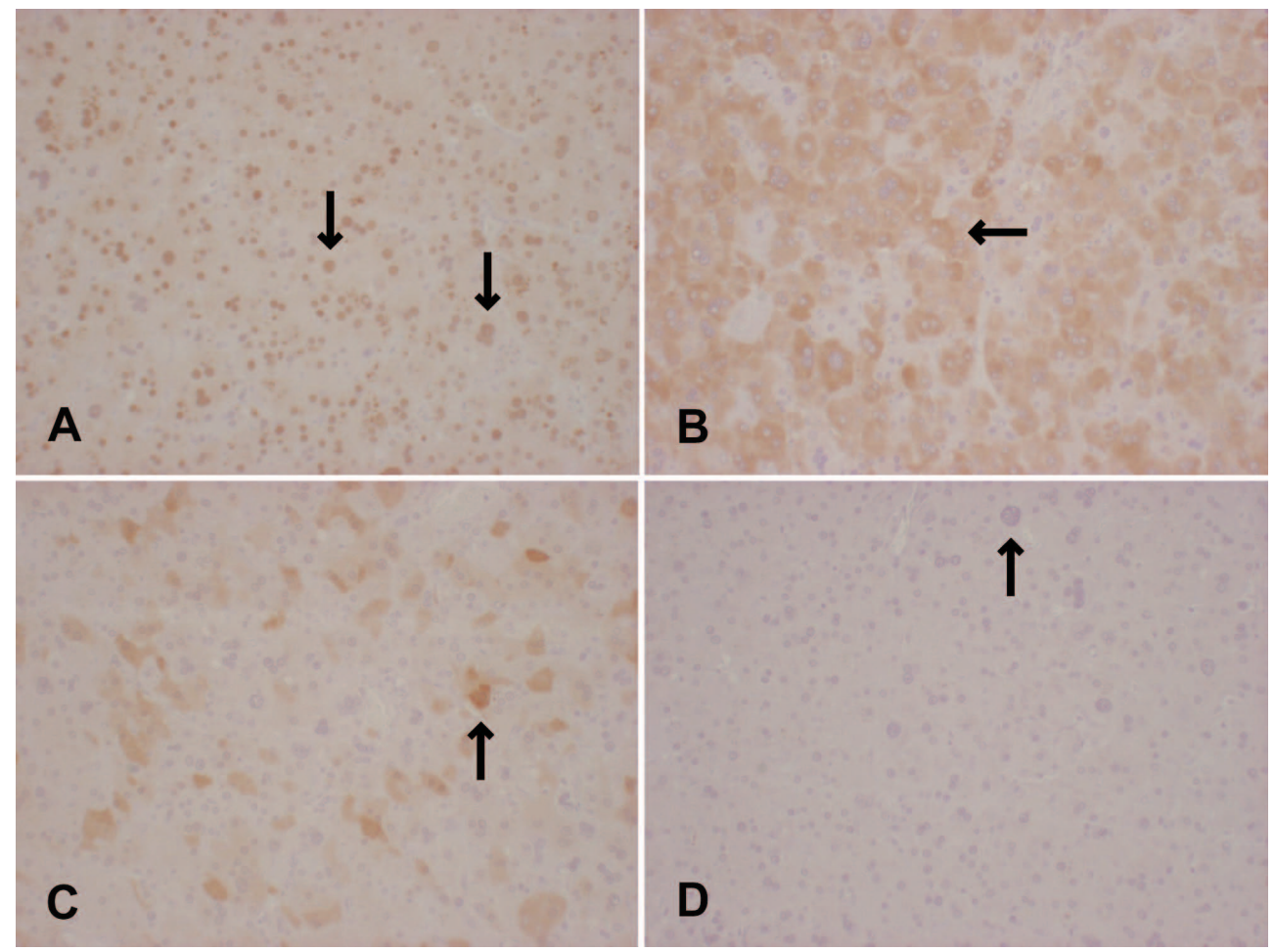

Fig. 3. Expression profiles of steroidogenic enzymes in the tumor with an original magnification $\times 200$. (A) Immunohistochemistry for steroidogenic factor-1 (SF-1). Nuclear immunoreactivity is detected in almost all of the tumor cells. Arrows represent the nuclear immunoreactivity of SF-1 in tumor cells. (B) Immunohistochemistry for $17 \alpha$-hydroxylase (P450c17). Immunoreactivity is mostly detected in the tumor cells. Arrow represents the positive immunoreactivity in the cytoplasm of tumor cells. (C) Immunohistochemistry for dehydroepiandrosterone sulfotransferase (DHEA-ST). Immunoreactivity is partially detected in the tumor cells. Arrow represents the positive immunoreactivity in the cytoplasm of tumor cells. (D) Immunohistochemistry for $3 \beta$-hydroxysteroid dehydrogenase ( $3 \beta$-HSD). Immunoreactivity is rarely detected in the tumor cells. Arrow represents the negative immunoreactivity in the cytoplasm of tumor cells.

adrenal rest or ectopic adrenal, the validity of applying the criteria of Weiss in these tumors has not been established at this juncture.

SF-1 is a universal regulator of steroidogenic P450 gene transcription and constantly expressed not only in the normal adrenal cortex but also in its hyperplasia or neoplasm (Arai et al. 2000). However, SF-1 can also be detected in the gonads, pituitary glands and placenta (Arai et al. 2000). P450c17 is involved in the synthesis of glucocorticoid and sex hormones, and is expressed only in the adrenal cortex and gonads (Arai et al. 2000). Immunoreactivity of DHEA-ST is detected in the adrenal cortex but not in the gonads (Arai et al. 2000). In our patient, SF-1 immunoreactivity was detected markedly in almost all of the tumor cells, and that for DHEA-ST was partially detected. These results all indicated that the tumor had characteristic of adrenocortical neoplasm. In addition, $3 \beta$-HSD is involved in the adrenal pathway of corticosteroid synthesis (Sasano et al. 1990). In our patient, the $3 \beta$-HSD immunoreactivity was rarely detected in the tumor cells. Therefore, results of immunohistochemical evaluation of these steroidogenic enzymes suggest that the tumor could produce adrenal androgens but not cortisol or aldosterone. This is also consistent with the patterns of disorga- nized steroidogenesis, characteristic of adrenocortical carcinoma (Sasano et al. 1993).

Malignant transformation of adrenal rest is extremely rare, as an especially non-functioning incidentally discovered (Goren et al. 1991; Arai et al. 2000). To the best of our knowledge, only several reports of ectopic adrenocortical carcinoma including the renal hilus, spinal region and testis, have been published in the literature (Goren et al. 1991; Bani-Hani 2003; Jain et al. 2008; Rodriguez et al. 2009; Akishima-Fukasawa et al. 2011). However, the detailed histopathological evaluations have been done only in one report (Akishima-Fukasawa et al. 2011) that presented Cushing's syndrome as usual. On the other hand, our patient had non-functioning tumor arising in the adrenal rest. The diagnosis of adrenocortical carcinoma arising from the adrenal rest is difficult because we do not know whether we could apply the criteria used in the primary adrenocortical neoplasms, and further investigations are required for clarification.

\section{Conflict of Interest}

All authors have no conflict of interest. 


\section{References}

Akishima-Fukasawa, Y., Yoshihara, A., Ishikawa, Y., Watanabe, N., Hiroi, N., Akasaka, Y., Sasano, H., Ishii, T. \& Yoshino, G. (2011) Malignant adrenal rest tumor of the retroperitoneum producing adrenocortical steroids. Endocr. Pathol., 22, 112-117.

Arai, K., Muro, H., Suzuki, M., Oba, N., Ito, K. \& Sasano, H. (2000) Adrenal rest tumor of the liver: a case report with immunohistochemical investigation of steroidogenesis. Pathol. Int., 50, 244-248.

Arola, J., Salmenkivi, K., Liu, J., Kahri, A.I. \& Heikkilä, P. (2000) p53 and Ki67 in adrenocortical tumors. Endocr. Res., 26, 861-865.

Bani-Hani, K.E. (2003) Primary non-functioning extra-adrenal adrenocortical carcinoma. Saudi. Med. J., 24, 301-304.

Goren, E., Engelberg, I.S. \& Eidelman, A. (1991) Adrenal rest carcinoma in hilum of kidney. Urology, 38, 187-190.

Icard, P., Goudet, P., Charpenay, C., Andreassian, B., Carnaille, B., Chapuis, Y., Cougard, P., Henry, J.F. \& Prove, C. (2001) Adrenocortical carcinomas: surgical trends and results of a 253-patient series from the French Association of Endocrine Surgeons study group. World J. Surg., 25, 891-897.

Jain, S.H., Sadow, P.M., Nosé, V. \& Dluhy, R.G. (2008) A patient with ectopic cortisol production derived from malignant testicular masses. Nat. Clin. Pract. Endoclinol. Metab., 4, 695-700.

Rodriguez, F.J., Scheithauer, B.W., Erickson, L.A., Jenkins, R.B. \& Giannini, C. (2009) Ectopic low-grade adrenocortical carcinoma in the spinal region: immunohistochemical and molecular cytogenetic study of a pediatric case. Am. J. Surg. Pathol., 33, 142-148.

Sasano, H., Mason, J.I., Sasano, N. \& Nagura, H. (1990) Immunolocalization of $3 \beta$-hydroxysteroid dehydrogenase in human adrenal cortex and in its disorders. Endocr. Pathol., 1, 94-101.

Sasano, H., Suzuki, T., Nagura, H. \& Nishikawa, T. (1993) Steroidogenesis in human adrenocortical carcinoma: biochemical activities, immunohistochemistry, and in situ hybridization of steroidogenic enzymes and histopathologic study in nine cases. Hum. Pathol., 24, 397-404.

Weiss, L.M. (1984) Comparative histologic study of 43 metastasizing and nonmetastasizing adrenocortical tumors. Am. J. Surg. Pathol., 8, 163-169. 